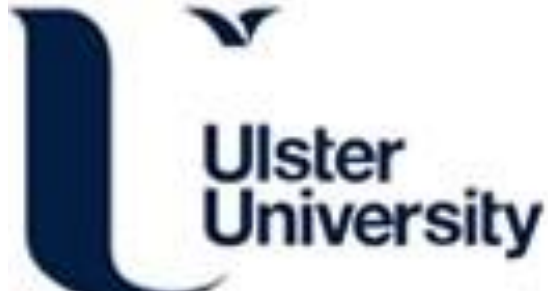

Energy Efficiency and Spectral Efficiency Trade-off for OFDM Systems with Imperfect Channel Estimation

Amin, O., Mohamed, E. B., Ahmed, M. H., \& Dobre, O. A. (2014). Energy Efficiency and Spectral Efficiency Trade-off for OFDM Systems with Imperfect Channel Estimation. 2014 IEEE International Conference on Communications (ICC). https://doi.org/10.1109/ICC.2014.6883872

Link to publication record in Ulster University Research Portal

Published in:

2014 IEEE International Conference on Communications (ICC)

Publication Status:

Published (in print/issue): 28/08/2014

DOI:

10.1109/ICC.2014.6883872

Document Version

Author Accepted version

\section{General rights}

Copyright for the publications made accessible via Ulster University's Research Portal is retained by the author(s) and / or other copyright owners and it is a condition of accessing these publications that users recognise and abide by the legal requirements associated with these rights.

\section{Take down policy}

The Research Portal is Ulster University's institutional repository that provides access to Ulster's research outputs. Every effort has been made to ensure that content in the Research Portal does not infringe any person's rights, or applicable UK laws. If you discover content in the Research Portal that you believe breaches copyright or violates any law, please contact pure-support@ulster.ac.uk. 


\title{
Energy Efficiency and Spectral Efficiency Trade-off for OFDM Systems with Imperfect Channel Estimation
}

\author{
Osama Amin ${ }^{1,2}$, Ebrahim Bedeer ${ }^{1}$, Mohamed H. Ahmed ${ }^{1}$, and Octavia A. Dobre ${ }^{1}$ \\ ${ }^{1}$ Faculty of Engineering and Applied Science, Memorial University of Newfoundland, \\ St. John's, NL, A1B 3X5, Canada. \\ E-mail: \{oamin, e.bedeer, mhahmed, odobre\}@mun.ca \\ ${ }^{2}$ Electrical Engineering Department, Faculty of Engineering, Assiut University, \\ Assiut, 71516, Egypt. \\ E-mail: amin@aun.edu.eg
}

\begin{abstract}
In this paper, the power loading problem for orthogonal frequency division multiplexing (OFDM) with imperfect channel estimation is investigated considering the trade-off between energy efficiency (EE) and spectral efficiency (SE). Unlike traditional research that uses the $\mathrm{EE}$ as an objective function and imposes constraints either on the SE or the achievable rate, we propound a multiobjective optimization approach that can flexibly switch between the $\mathrm{EE}$ and the $\mathrm{SE}$ functions or change the priority level of each function using a trade-off parameter. Our dynamic approach is more tractable than conventional approaches and more convenient to realistic communication applications and scenarios. The system model considers the path loss and shadowing effect in modeling the EE and SE metrics, in addition to taking the channel estimation error into account. We first solve the marginal problems of maximizing the $\mathrm{EE}$ and the SE individuality, and then prove that the multiobjective optimization of the $\mathrm{EE}$ and the $\mathrm{SE}$ is equivalent to a simple problem that maximizes the capacity and minimizes the total power consumption. Finally, we use numerical results to discuss the choice of the trade-off parameter and study the effect of the estimation error, transmission power budget and channel-to-noise ratio on the multiobjective optimization.
\end{abstract}

\section{INTRODUCTION}

The dramatic growth of wireless communication services and applications represents the main driving force to expand the existing wireless infrastructure and deploy new systems. The tremendous energy consumption at the base stations accounts for the most of energy consumption of cellular networks, which represents an apparent contribution of the information and communication technology industry to the global $\mathrm{CO}_{2}$ emissions [1]. Therefore, wireless communication systems have to be designed based on green metrics that reduce the energy consumption wisely, along with the associated $\mathrm{CO}_{2}$ emissions.

Energy efficiency (EE) is a widely used green communication metric, defined as the number of successfully delivered bits per unit energy or its inverse, i.e., the total energy consumption to deliver one bit correctly [2]. Although EE is the major design metric for environment-friendly future wireless communication systems, it conflicts with other traditional metrics such as spectral efficiency (SE) [3]. The trade-off relation between EE and SE states that the available system's resources cannot be optimized to improve EE and SE simultaneously. To tackle such cases, optimization problems are formulated to improve EE under SE or channel capacity constraints for single or multiple users in [4]-[7].

The previously mentioned resource allocation problems that deal with the EE-SE trade-off, target to fix the EE as the objective function and impose a constraint on SE according to the usage limitations. On the other hand, there are scenarios in wireless communications systems in which we need to change the optimization objective function according to the surrounding circumstances or the application requirements. A motivating scenario is when renewable energy sources are used in addition to diesel generators in base stations to generate the required electrical energy [8]. In such a scenario, when the base station is powered by clean energy sources (i.e., renewable sources), adopting EE as a design metric does not have a green advantage, as the base station is working now with zero $\mathrm{CO}_{2}$ emissions. Thus, it is more beneficial to improve other quality of services (QoS) metrics, such as SE and delay. On the other hand, when the surrounding environment varies, diesel generators are used to compensate for the shortage of the renewable sources or even replace them. Therefore, considering the EE as the objective function becomes inevitable when diesel generators is the only energy source, while a multiobjective function of both EE and SE is used when both sources are used together. In the later case, the priority of each function is chosen according to the contribution of each energy source in powering the base station. Another motivating scenario is the everlasting variation of the service requirements with each user, such as real time, non-real time and rate-hungry applications. For real time applications, the transmission rate should be above a given value that depends on the type of data, and maximizing the $\mathrm{EE}$ subject to SE constraint becomes a suitable optimization problem. For the non-real time applications, we mainly focus on maximizing EE. On the other hand, for rate-hungry applications, the SE becomes the main objective function with possible consideration to EE. The aforementioned different cases can be integrated in a multiobjective optimization problem of EE and SE with a trade-off parameter that adjusts the priority of each function according to the application type and QoS requirements. 
Motivated by the previously discussed examples, the multiobjective optimization becomes the key method to consider conflicting objective functions and introduce a solution for a given priority of each function [9], [10]. The multiobjective optimization introduces flexible solutions suitable to different practical scenarios, with a lower complexity when compared with solving several single objective optimization problems and switching between their solutions. In this paper, inspired by the green communication trend and considering the variations of the objective functions according to the provided service or the surrounding environment, we investigate the multiobjective optimization of EE and SE for the OFDM system with imperfect channel state information (CSI). We assume using linear minimum mean square error (LMMSE) for channel estimation, with the estimation error inversely proportional to the signal-to-noise ratio (SNR) [11]. We first solve the marginal problems of EE and SE assuming imperfect CSI and then prove that the multiobjective optimization of maximizing both $\mathrm{EE}$ and $\mathrm{SE}$ is equivalent to the multiobjective optimization of minimizing the total power consumption and maximizing the channel capacity. Finally, we employ the simplified multiobjective optimization form to find the adaptive power loading solutions for OFDM systems with imperfect CSI assuming total power constraint.

The rest of the paper is organized as follows. In Section II, we describe the system model and formulate the EE and SE metrics in imperfect CSI. In Section III, we find the optimal power loading for maximum EE and SE, then we solve the multiobjective optimization problem of EE and SE for a given trade-off parameter. Then, we present numerical simulation in Section IV and conclude the paper in Section V.

\section{System Model AND ENERGy EFFICIENCY METRIC}

In this section, we introduce the model of the OFDM system with LMMSE channel estimation and define the EE and SE metrics. We assume a serial data sequence that is divided into blocks, processed by a given precoded matrix and loaded to $M$ subcarriers. The channel is assumed to change slowly and is modeled as a finite impulse response system with order equal to $L, \mathbf{h}=[h(0), h(1), \cdots, h(L)]^{T}$, where each channel tap is assumed to be complex Gaussian distributed with zeromean and variance $\sigma_{h}^{2}$. To avoid the inter-block interference, a cyclic prefix is added at the transmitter and removed at the receiver. The noise at the receive-side is modeled as additive white Gaussian noise (AWGN) with zero mean and correlation matrix equal to $\sigma_{n}^{2} \mathbf{I}$. The training pilot symbols $\mathbf{b}$ are added to the precoded block, where the receiver knows the pilot pattern and estimates the channel using the LMMSE estimator as [11]

$$
\hat{\mathbf{h}}=\left(\sigma_{n}^{2} \mathbf{R}_{h}^{-1}+\mathbf{B}^{H} \mathbf{B}\right)^{-1} \mathbf{B}^{H} \mathbf{x}
$$

where $\mathbf{B}$ is an $M \times(L+1)$ column wise circulant matrix with the first column equal to $\mathbf{b}$. The subchannel estimates are computed as [11]

$$
\left[\hat{H}(1), \hat{H}(W), \ldots, \hat{H}\left(W^{M-1}\right)\right]^{T}=\sqrt{M} \mathbf{F}_{L} \hat{\mathbf{h}}
$$

where $W=\exp (j 2 \pi / M), \mathbf{F}_{L}$ is the first $L+1$ submatrix of $\mathbf{F}$, and $\mathbf{F}$ is the $M \times M$ discrete Fourier transform (DFT) matrix with the $(m, n)$ element defined $[\mathbf{F}]_{m, n}=W^{-m n} / \sqrt{M}$.
The channel capacity is expressed in terms of the channel estimate across subcarriers similarly to [11], while considering powers per subcarriers $\left(\mathbf{P}=\left[P_{1}, P_{2}, \ldots, P_{M}\right]^{T}\right)$,

$$
C(\mathbf{P})=B \sum_{m=1}^{M} \log _{2}\left(1+\frac{\left|\hat{H}\left(W^{m}\right)\right|^{2} G P_{m}}{\sigma_{\Delta H}^{2} G P_{m}+\sigma_{n}^{2}}\right),
$$

where $B$ is the subcarrier bandwidth, $P_{m}$ is the $m$ th subcarrier power, $G$ is the large scale fading power coefficient, and $\sigma_{\Delta H}^{2}$ is the minimum mean square error (MMSE). The latter can be expressed as [11]

$$
\sigma_{\Delta H}^{2}=\frac{(L+1) \sigma_{h}^{2} \sigma_{n}^{2}}{\sigma_{n}^{2}+\sigma_{h}^{2} G P_{\mathrm{p}}},
$$

where $P_{\mathrm{p}}$ is the pilots' transmitted power, and the noise variance is defined as $\sigma_{n}^{2}=N_{0} N_{\mathrm{f}} B$, with $N_{0}$ as the noise power spectral density and $N_{\mathrm{f}}$ as the noise figure. The power loss due to the large scale fading captures both the path loss and shadowing loss, and can be expressed in $\mathrm{dB}$ as [12],

$$
G_{\mathrm{Loss}, \mathrm{dB}}=G_{\mathrm{PL}, 0}+10 n \log _{10}\left(d / d_{0}\right)+S,
$$

where $G_{\mathrm{PL}, 0}$ is the path loss at a reference distance $d_{0}, d$ is the distance between the transmitter and receiver, $n$ is the exponent loss, and $S$ is the shadowing fading power loss in $\mathrm{dB}$ and is modeled as a zero-mean Gaussian random variable with a standard deviation $\sigma_{\mathrm{s}}$.

\section{The EE of the OFDM is expressed as}

$$
\eta_{\mathrm{EE}}=\frac{C(\mathbf{P})}{\kappa \sum_{m=1}^{M} P_{m}+P_{\mathrm{c}}},
$$

where $\kappa$ is a constant depending on the power amplifier efficiency and $P_{\mathrm{c}}$ is the circuitry power consumption. The SE of the OFDM system with LMMSE channel estimation is expressed as

$$
\eta_{\mathrm{SE}}=\frac{C(\mathbf{P})}{B}=\sum_{m=1}^{M} \log _{2}\left(1+\frac{\left|\hat{H}\left(W^{m}\right)\right|^{2} G P_{m}}{\sigma_{\Delta H}^{2} G P_{m}+\sigma_{n}^{2}}\right) .
$$

\section{EE AND SE POWER LOADING SCHEMES}

In this section, we aim to find the power loading solution that improves the conflicting objective functions (i.e., EE and SE) individually, then jointly as a multiobjective optimization approach.

\section{A. Optimal Power Loading for Maximum EE}

The optimal power loading solution that maximizes the EE can be found by solving the equivalent optimization problem that minimizes the inverse of $\mathrm{EE}$,

$$
\begin{array}{ll}
\min _{m=1,2, \cdots M} & \eta_{\mathrm{EE}}^{-1}=\frac{\kappa \sum_{m=1}^{M} P_{m}+P_{\mathrm{c}}}{C(\mathbf{P})} \\
\text { subject to } & \sum_{m=1}^{M} P_{m} \leq P_{\mathrm{T}} .
\end{array}
$$


By following [13], the nonlinear fractional optimization problem in (8) is transformed to the following equivalent parameterized convex function

$$
\mathcal{L}_{\mathrm{EE}}=\kappa \sum_{m=1}^{M} P_{m}+P_{\mathrm{c}}-q C(\mathbf{P}),
$$

where the parameter $q$ is a constant equal to the minimum value of our objective function $\left(\eta_{\mathrm{EE}}^{-1}\right)$ and is computed such that $\mathcal{L}_{E E}(q)=0$. Based on (9), the optimization problem is formulated as,

$$
\begin{aligned}
\min _{P_{m}, m=1,2, \cdots M} & \mathcal{L}_{\mathrm{EE}} \\
\text { subject to } & \sum_{m=1}^{M} P_{m} \leq P_{\mathrm{T}} .
\end{aligned}
$$

The constrained optimization problem in (10) can be expressed in the Lagrangian form as

$$
\begin{aligned}
\mathcal{L}_{\mathrm{EE}, \text { con }}= & \kappa \sum_{m=1}^{M} P_{m}+P_{\mathrm{c}} \\
& -q B \sum_{m=1}^{M} \log _{2}\left(1+\frac{\left|\hat{H}\left(W^{m}\right)\right|^{2} G P_{m}}{\sigma_{\Delta H}^{2} G P_{m}+\sigma_{n}^{2}}\right) \\
& +\lambda\left(\sum_{m=1}^{M} P_{m}-P_{\mathrm{T}}+y^{2}\right),
\end{aligned}
$$

where $\lambda$ is the Lagrange multiplier corresponding to the total power constraint and $y^{2}$ is the slack variable referring to the required inequality in (10). By differentiating $\mathcal{L}_{\mathrm{EE} \text {,con }}$ with respect to $\lambda, y$ and $P_{m}, m=1,2, \ldots, M$, and equating the results with zero, one obtains

$$
\begin{gathered}
\left(\sigma_{\Delta H}^{2} P_{m}+\sigma_{\mathrm{n}}^{2}\right)\left(\left(\sigma_{\Delta H}^{2}+\left|\hat{H}\left(W^{m}\right)\right|^{2} G\right) P_{m}+\sigma_{n}^{2}\right) \\
-\left(\frac{q B}{\ln 2}\right) \frac{\left|\hat{H}\left(W^{m}\right)\right|^{2} G \sigma_{n}^{2}}{(\kappa+\lambda)}=0 \\
\sum_{m=1}^{M} P_{m}-P_{\mathrm{T}}+y^{2}=0 \\
2 \lambda y=0 .
\end{gathered}
$$

From (14), we have two possible scenarios: either $\lambda=0$ or $y=0$. Assume first that $\lambda=0$, which refers to the validity of the total power constraint. In this case, the power loading coefficients are calculated from (12) as

$$
P_{\mathrm{EE}, m}=\rho_{m}\left[-1+\sqrt{1+\frac{2\left(\frac{q B}{\kappa \ln 2}\left|\hat{H}\left(W^{m}\right)\right|^{2}-\frac{\sigma_{n}^{2}}{G}\right)}{\left(2 \sigma_{\Delta H}^{2}+\left|\hat{H}\left(W^{m}\right)\right|^{2}\right) \rho_{m}}}\right]_{(15)}^{+},
$$

where $[x]^{+}$denotes $\max (0, x)$ and $\rho_{m}$ is expressed as

$$
\rho_{m}=\frac{\sigma_{\mathrm{n}}^{2}\left(2 \sigma_{\Delta H}^{2}+\left|\hat{H}\left(W^{m}\right)\right|^{2}\right)}{2 \sigma_{\Delta H}^{2}\left(\sigma_{\Delta H}^{2}+\left|\hat{H}\left(W^{m}\right)\right|^{2}\right) G} .
$$

The constant coefficient $q$ is computed by setting equation (9) equal to zero and using the subcarrier's power solution found in (15) as,

$$
\begin{aligned}
& \kappa \sum_{m=1}^{M} P_{\mathrm{EE}, m}+P_{\mathrm{c}} \\
& -q B \sum_{m=1}^{M} \log _{2}\left(1+\frac{\left|\hat{H}\left(W^{m}\right)\right|^{2} G P_{\mathrm{EE}, m}}{\sigma_{\Delta H}^{2} G P_{\mathrm{EE}, m}+\sigma_{n}^{2}}\right)=0 .
\end{aligned}
$$

After finding $q$ from (17), we compute $P_{\mathrm{EE}, m}$ from (15) and check the total power constraint. If the constraint is verified, then we obtained the required solution. On the other hand, if the constraint is violated, then we switch to the second scenario of (14), which assumes $y=0$. In such case, the power of the $m$ th subcarrier is expressed in terms of the Lagrange Multiplier $(\lambda)$ as

$$
P_{\mathrm{EE}, m}=\rho_{m}\left[-1+\sqrt{1+2 \frac{\frac{q B}{(\kappa+\lambda) \ln 2}\left|\hat{H}\left(W^{m}\right)\right|^{2}-\frac{\sigma_{n}^{2}}{G}}{\left(2 \sigma_{\Delta H}^{2}+\left|\hat{H}\left(W^{m}\right)\right|^{2}\right) \rho_{m}}}\right]^{+}
$$

The constants $q$ and $\lambda$ are found by replacing (18) in (13) and $\mathcal{L}_{\mathrm{EE}, \text { con }}=0$ at $y=0$ as discussed in [13].

\section{B. Optimal Power Loading for Maximum SE}

In this subsection, we solve the optimal power loading that maximizes the spectral efficiency under the total power constraint, which is formulated as

$$
\begin{aligned}
\max _{P_{m}, m=1,2, \cdots M} & \eta_{\mathrm{SE}} \\
\text { subject to } & \sum_{m=1}^{M} P_{m} \leq P_{\mathrm{T}} .
\end{aligned}
$$

The constrained optimization problem is expressed in the Lagrangian form as

$$
\begin{array}{r}
\mathcal{L}_{\mathrm{SE}, \text { con }}=\sum_{m=1}^{M} \log _{2}\left(1+\frac{\left|\hat{H}\left(W^{m}\right)\right|^{2} G P_{m}}{\sigma_{\Delta H}^{2} G P_{m}+\sigma_{n}^{2}}\right) \\
+\lambda\left(P_{\mathrm{T}}-\sum_{m=1}^{M} P_{m}+y^{2}\right) .
\end{array}
$$

By finding the derivatives of $\mathcal{L}_{\mathrm{SE} \text {,con }}$ with respect to $\lambda, y$ and $P_{m}, m=1,2, \ldots, M$, and equating them to zero, the solutions are found to exist only at $y=0$ since the objective function is monotonically increasing. Thus, one can show that the solution of the $m$ th subcarrier's power is

$$
P_{\mathrm{SE}, m}=\rho_{m}\left[-1+\sqrt{1+2 \frac{\left(\frac{1}{\lambda \ln 2}\right)\left|\hat{H}\left(W^{m}\right)\right|^{2}-\frac{\sigma_{n}^{2}}{G}}{\left(2 \sigma_{\Delta H}^{2}+\left|\hat{H}\left(W^{m}\right)\right|^{2}\right) \rho_{m}}}\right]^{+},
$$

where $\lambda$ is computed from the total power constraint equation, i.e., by replacing (21) in (13) at $y=0$. 
C. Adaptive Power Loading for EE-SE Multiobjective Optimization

Optimizing EE or SE under a given set of constraints is suitable for a specific communication scenario/application or under static environmental circumstances. On the hand, the realistic conditions change dynamically, and changing the objective function becomes indispensable. In the following analysis, we consider a multiobjective optimization problem that aims to maximize both $\mathrm{EE}$ and SE through minimizing the inverse of $\mathrm{EE}$ and inverse of $\mathrm{SE}$ as

$$
\begin{aligned}
& \min _{P_{m}} \eta_{\mathrm{EE}}^{-1}\left(P_{m}\right) \quad \text { and } \quad \min _{P_{m}} \eta_{\mathrm{SE}}^{-1}\left(P_{m}\right) \\
& \text { subject to } \quad \sum_{m=1}^{M} P_{m} \leq P_{\mathrm{T}} .
\end{aligned}
$$

The multiobjective function is expressed using the trade-off parameter, $\beta$, with $0<\beta<1$, which describes the priority level of each single objective function as follows,

$$
\begin{aligned}
\mathcal{L}_{\mathrm{EE}-\mathrm{SE}} & =\beta \theta_{\mathrm{EE}} \eta_{\mathrm{EE}}^{-1}+(1-\beta) \theta_{\mathrm{SE}} \eta_{\mathrm{SE}}^{-1} \quad 0<\beta<1 \\
& =\beta \frac{\theta_{\mathrm{EE}}\left(\kappa \sum_{m=1}^{N} P_{m}+P_{\mathrm{c}}\right)}{C(\mathbf{P})}+(1-\beta) \frac{\theta_{\mathrm{SE}} B}{C(\mathbf{P})},
\end{aligned}
$$

where $\theta_{\mathrm{EE}}$ and $\theta_{\mathrm{SE}}$ are normalized factors used for both objective functions in order to have a similar scale. Similar to (10) and (12), we transform the fractional objective function $\left(\mathcal{L}_{\mathrm{EE}-\mathrm{SE}}\right)$ in $(23)$ to a parameterized convex problem as,

$$
\begin{aligned}
\mathcal{L}_{\mathrm{EE}-\mathrm{SE}, 1}=\beta \theta_{\mathrm{EE}}\left(\kappa \sum_{m=1}^{N} P_{m}+P_{\mathrm{c}}\right) & +(1-\beta) \theta_{\mathrm{SE}} B \\
& -q_{1} C(\mathbf{P}),
\end{aligned}
$$

where $q_{1}$ is a constant parameter that represents the minimum value of (23). The term $(1-\beta) \theta_{\mathrm{SE}} B$ in (24) is a constant, thus omitting it does not affect the problem solution, and the following objective is used instead,

$$
\mathcal{L}_{\mathrm{EE}-\mathrm{SE}, 2}=\beta \theta_{\mathrm{EE}}\left(\kappa \sum_{m=1}^{N} P_{m}+P_{\mathrm{c}}\right)-q_{1} C(\mathbf{P}) .
$$

Dividing the the objective function $\mathcal{L}_{\mathrm{EE}-\mathrm{SE}, 2}$ by $\beta \theta_{\mathrm{EE}}$ gives another objective function that has the same solution and is expressed as

$$
\mathcal{L}_{\mathrm{EE}-\mathrm{SE}, 3}=\left(\kappa \sum_{m=1}^{N} P_{m}+P_{\mathrm{c}}\right)-\frac{q_{1}}{\beta \theta_{\mathrm{EE}}} C(\mathbf{P}) .
$$

The quantity $q_{1} /\left(\beta \theta_{\mathrm{EE}}\right)$ is a positive constant and is equivalently replaced by $(1-\alpha) / \alpha$, where $0<\alpha<1$,

$$
\mathcal{L}_{\mathrm{EE}-\mathrm{SE}, 4}=\left(\kappa \sum_{m=1}^{N} P_{m}+P_{\mathrm{c}}\right)-\frac{(1-\alpha)}{\alpha} C(\mathbf{P}) .
$$

Multiplying the objective function in (27) by $\alpha$ yields an equivalent objective function that is written as

$$
\begin{aligned}
& \mathcal{L}_{\mathrm{EE}-\mathrm{SE}, 5}=\alpha\left(\kappa \sum_{m=1}^{N} P_{m}+P_{\mathrm{c}}\right)-(1-\alpha) C(\mathbf{P}) \\
& \equiv \min _{P_{m}}\left(\kappa \sum_{m=1}^{N} P_{m}+P_{\mathrm{c}}\right) \text { and } \max _{P_{m}} C(\mathbf{P}) .
\end{aligned}
$$

The objective function written in (28) represents a multiobjective function that aims to minimize the total power consumption and maximize the channel capacity using the tradeoff parameter $\alpha$, as presented in (29). Thus, the joint optimization problem that targets to maximize the EE and SE is equivalent to minimizing the total power consumption and maximizing the channel capacity. Based on this proved fact, the optimization of (23) can be equivalently written as,

$$
\begin{array}{ll}
\min _{P_{m}} & \frac{\alpha}{\theta_{\mathrm{P}}}\left(\kappa \sum_{m=1}^{N} P_{m}+P_{\mathrm{c}}\right)-\frac{(1-\alpha)}{\theta_{\mathrm{C}}} C(\mathbf{P}) \\
\text { subject to } & \sum_{m=1}^{M} P_{m} \leq P_{\mathrm{T}}
\end{array}
$$

where $\theta_{\mathrm{P}}$ and $\theta_{\mathrm{C}}$ are the normalized factor for the power and the capacity objective functions, respectively. The multiobjective problem in (30) reduces to capacity or SE maximization at $\alpha=0$, and to power minimization at $\alpha=1$. The energy efficient solution is achieved at $\alpha_{\mathrm{EE}}$ that will be computed later on in the paper. The Lagrangian problem of (30) is written as,

$$
\begin{aligned}
\mathcal{L}_{\mathrm{EE}-\mathrm{SE}, \mathrm{con}}= & \frac{\alpha}{\theta_{\mathrm{P}}}\left(\kappa \sum_{m=1}^{N} P_{m}+P_{\mathrm{c}}\right)-\frac{(1-\alpha) B}{\theta_{\mathrm{C}}} C(\mathbf{P}) \\
& +\lambda\left(\sum_{m=1}^{M} P_{m}-P_{\mathrm{T}}+y^{2}\right)
\end{aligned}
$$

By differentiating $\mathcal{L}_{\mathrm{EE}-\mathrm{SE} \text {,con }}$ with respect to $\lambda, y$ and $P_{m}$, $m=1,2, \ldots, M$, and equating the results with zero, we obtain the following equations,

$$
\begin{gathered}
\left(\sigma_{\Delta H}^{2} P_{m}+\sigma_{n}^{2}\right)\left(\left(\sigma_{\Delta H}^{2}+\left|\hat{H}\left(W^{m}\right)\right|^{2} G\right) P_{m}+\sigma_{n}^{2}\right) \\
-\left(\frac{\theta_{\mathrm{C}}(1-\alpha) B}{\left(\theta_{\mathrm{P}} \alpha \kappa+\lambda \theta_{\mathrm{C}}\right) \ln 2}\right)\left|\hat{H}\left(W^{m}\right)\right|^{2} G \sigma_{n}^{2}=0 \\
\sum_{m=1}^{M} P_{m}-P_{\mathrm{T}}+y^{2}=0 \\
2 \lambda y=0 .
\end{gathered}
$$

According to equation (34) and similar to the solution for EE optimization in Section III-A, we assume $\lambda=0$, which imposes the validity of the total power constraint and obtain the power of the $m$ th subcarrier from (32) as

$P_{\mathrm{EE}-\mathrm{SE}, m}=\rho_{m}\left[\sqrt{1+\frac{2\left(\frac{(1-\alpha) B\left|\hat{H}\left(W^{m}\right)\right|^{2}}{\left(\theta_{\mathrm{C}} / \theta_{\mathrm{P}}\right) \alpha \kappa \ln 2}-\frac{\sigma_{n}^{2}}{G}\right)}{\rho_{m}\left(2 \sigma_{\Delta H}^{2}+\left|\hat{H}\left(W^{m}\right)\right|^{2}\right)}}-1\right]$.

By checking the total power constraint using the solution given in (35), we impose $y=0$ if the constraint is violated, and the power of the $m$ th subcarrier is expressed in terms of $\lambda$ as,

$P_{\mathrm{EE}-\mathrm{SE}, m}=\rho_{m}\left[\sqrt{1+\frac{2\left(\mu\left|\hat{H}\left(W^{m}\right)\right|^{2}-\frac{\sigma_{n}^{2}}{G}\right)}{\rho_{m}\left(2 \sigma_{\Delta H}^{2}+\left|\hat{H}\left(W^{m}\right)\right|^{2}\right)}}-1\right]^{+}$, 
TABLE I: Simulation Parameters.

\begin{tabular}{|c|c|c|}
\hline$P_{\mathrm{c}}=300 \mathrm{~W}$ & $\kappa=7.8$ & $L=5$ \\
\hline$B_{\text {total }}=1.25 \mathrm{MHz}$ & $M=128$ & $S=13.2 d B$ \\
\hline$d_{0}=1 \mathrm{~m}$ & $d=1050 \mathrm{~m}$ & $G_{\mathrm{PL}, 0}=-80.4 d B$ \\
\hline$N_{\mathrm{f}}=7 \mathrm{~dB}$ & $N_{0}=-171 \mathrm{dBm} / \mathrm{Hz}$ & $n=3.5$ \\
\hline
\end{tabular}

where $\lambda$ is found from (33) with $y=0$, and $P_{m}=P_{\mathrm{EE}-\mathrm{SE}, m}$ and $\mu$ is expressed as

$$
\mu=\left(\frac{\theta_{\mathrm{P}}(1-\alpha) B}{\theta_{\mathrm{C}}\left(\alpha \kappa+\lambda \theta_{\mathrm{P}}\right) \ln 2}\right)
$$

It is noteworthy that the trade-off parameter $\alpha$ is adjusted to obtain a required priority of the objective functions. To optimize the SE objective function, we impose $\alpha=0$, while to optimize $\mathrm{EE}$, we use $\alpha_{\mathrm{EE}}$ that can be found from $\mathcal{L}_{\mathrm{EE}-\mathrm{SE}, \mathrm{con}}=0$, i.e., similar to finding $q$ in solving the $\mathrm{EE}$ problem. On the other hand, for a specific priority to EE or $\mathrm{SE}, \alpha$ is chosen according to the average performance that is discussed in the next section ${ }^{1}$.

\section{Simulation Results}

In this section, we provide a numerical evaluation of the proposed multiobjective optimization problem for an OFDM system with imperfect channel estimation. The simulation parameters are considered for mobile Worldwide Interoperability for Microwave Access (WiMAX) base stations and summarized in Table I using a single antenna for the transmission [14]. The normalization factors used in our objective function are assumed to be $\theta_{\mathrm{P}}=\kappa P_{\mathrm{T}}+P_{\mathrm{C}}$ and $\theta_{\mathrm{C}}=3.6 \mathrm{Mb} / \mathrm{s}$ [14].

Simulation Example 1: In the first simulation example, we study the EE and SE behavior versus $\alpha$ for different ratios of maximum transmitted power and maximum total power consumption $\left(P_{T} / \theta_{\mathrm{P}}\right)$ in Fig. 1 . We assume the channel-tonoise ratio $(\mathrm{CNR})$ is $20 \mathrm{~dB}$ and the MMSE of the channel estimator equal to 0.001 . First, we aim to investigate the choice of the trade-off parameter $\alpha$ to achieve a given priority level for either EE or SE. For $\alpha=0$ the solution reduces to maximum $\mathrm{SE}$ or capacity, and for $\alpha=1$, the scenario reduces to the minimum transmitted power. On the other hand, maximum average $\mathrm{EE}$ is achieved at $\alpha_{\mathrm{EE}}=0.5$. As alpha increases from zero to $\alpha_{\mathrm{EE}}$, i.e., our region of interest, the $\mathrm{EE}$ increases and SE decreases, while both EE and SE decrease for $\alpha=\alpha_{\mathrm{EE}}$ to 1 . The average results depicted in Figure 1 are used as a guide to choose $\alpha$ in order to achieve a predetermined average $\mathrm{EE}$ or SE performance. For example, for $P_{T} / \theta_{\mathrm{P}}=0.11$, if it is required to design the system for a green perspective while satisfying average $\mathrm{SE}$ to $0.6 \mathrm{kbit} / \mathrm{sec} / \mathrm{Hz}$, then we choose $\alpha=0.3$, while if the design targets to maximize the spectral efficiency while keeping minimum average $\mathrm{EE}$ to $4 \mathrm{~kb} / \mathrm{J}$, then we choose $\alpha=0.1$. As noted from this example, we can switch between different optimization problem by changing the priority factor and use the same solution rather than solving different optimization problems.

Additionally, we aim to study the effect of the maximum useful transmission power budget ratio to the maximum total power consumption at the base station, i.e. $\left(P_{T} / \theta_{\mathrm{P}}\right)$. For large transmitted power budget $\left(P_{T} / \theta_{\mathrm{P}}=0.11\right)$, we observe the

\footnotetext{
${ }^{1}$ It is worthy to mention that the solutions of our optimization problems satisfy the Karush-Kuhn-Tucker conditions; the proof is not provided due to space limitations
}

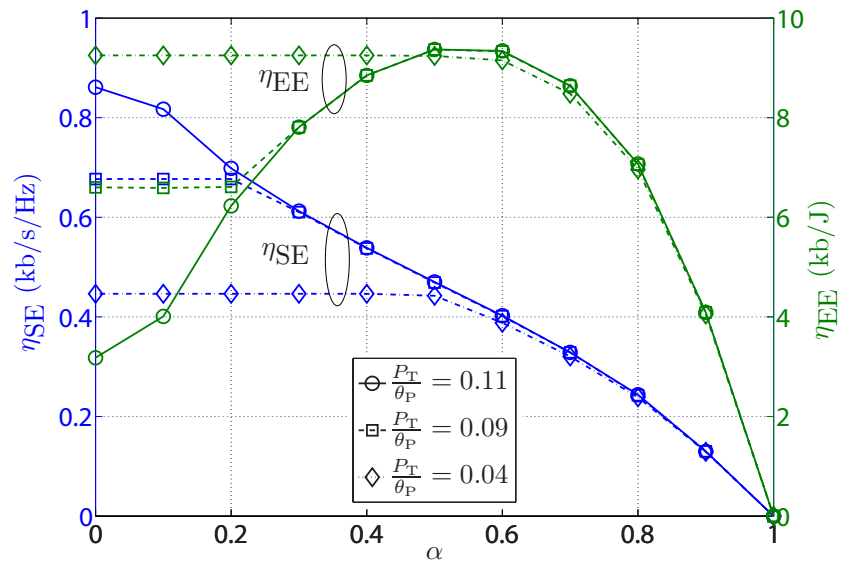

Fig. 1: EE and SE versus the priority parameter $\alpha$ for different $P_{T} / \theta_{\mathrm{P}}$ values.

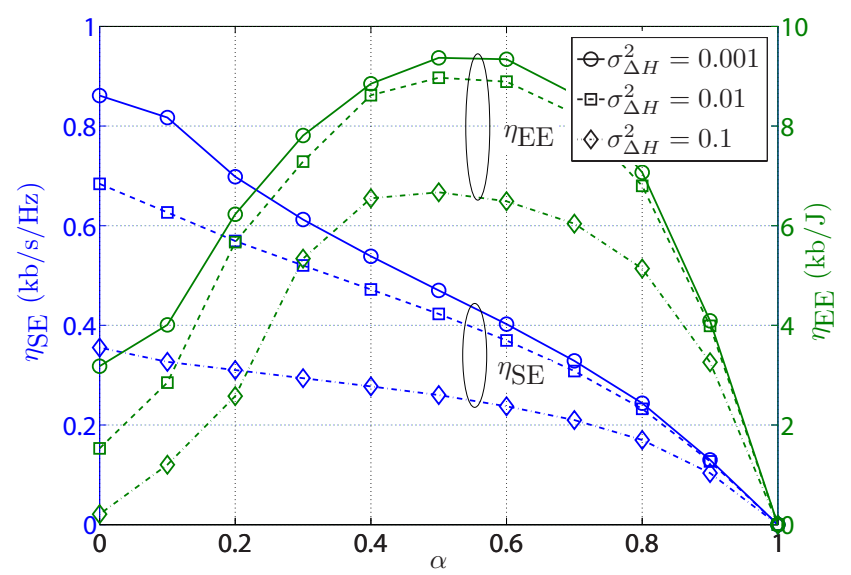

Fig. 2: EE and SE versus the priority parameter $\alpha$ for different MMSE values and at $P_{T} / \theta_{\mathrm{P}}=0.11$.

expected trade-off relation between EE and SE versus $\alpha$. On the other hand, the unavailability of sufficient transmission power budget $\left(P_{T} / \theta_{\mathrm{P}}=0.04\right)$ limits the $\mathrm{SE}$ from improving as $\alpha$ decreases and limits the EE curve from its expected trend, i.e., having a maximum at $\alpha_{\mathrm{EE}}$.

Simulation Example 2: To study the effect of the channel estimation error on the trade-off relation between EE and SE, in Fig. 2, we plot the EE and SE curves versus $\alpha$ for $P_{T} / \theta_{\mathrm{P}}=$ 0.11 and assuming different MMSE values. The performance trend of EE and SE is the same for different estimation quality, but with a gain loss as the MMSE increases. The loss between curves decreases as MMSE decreases. MMSE significantly affects the maximum SE and EE solutions, respectively. More specifically, EE reduces around $30 \%$ when the MMSE equals to 0.1 when compared with MMSE equal to 0.001. On the other hand, maximum SE shows increased sensitivity to MMSE, as the SE reduces around $40 \%$ at MMSE equal to 0.1 when compared with MMSE equals to 0.001.

Simulation Example 3: In the third simulation example, we focus on the EE and SE cases that change significantly with MMSE. These cases represent the optimum EE solution (at $\alpha_{\mathrm{EE}}$ ), its corresponding SE, as well as the optimal SE solution (at $\alpha=0$ ) and its corresponding EE. Fig. 3 shows the relation 


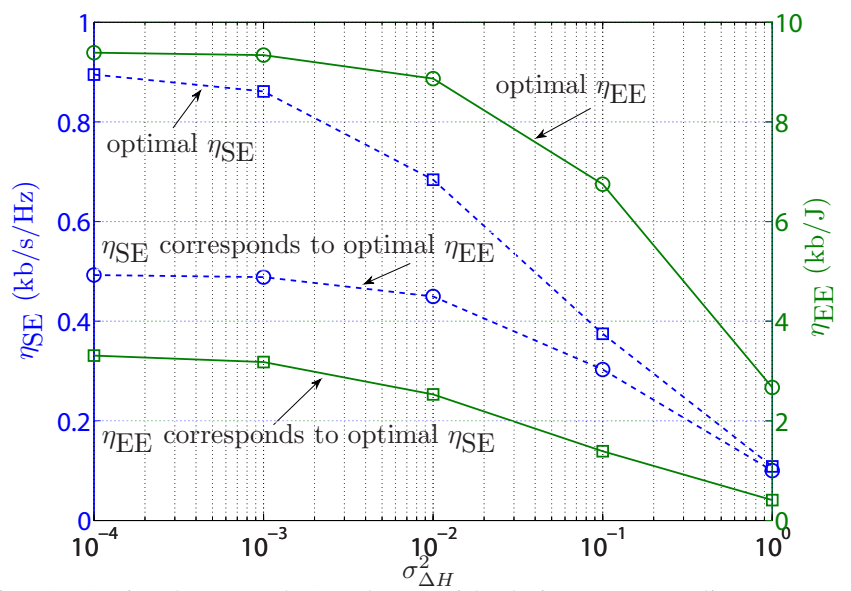

Fig. 3: Optimal EE and SE along with their corresponding SE and EE, versus MMSE.

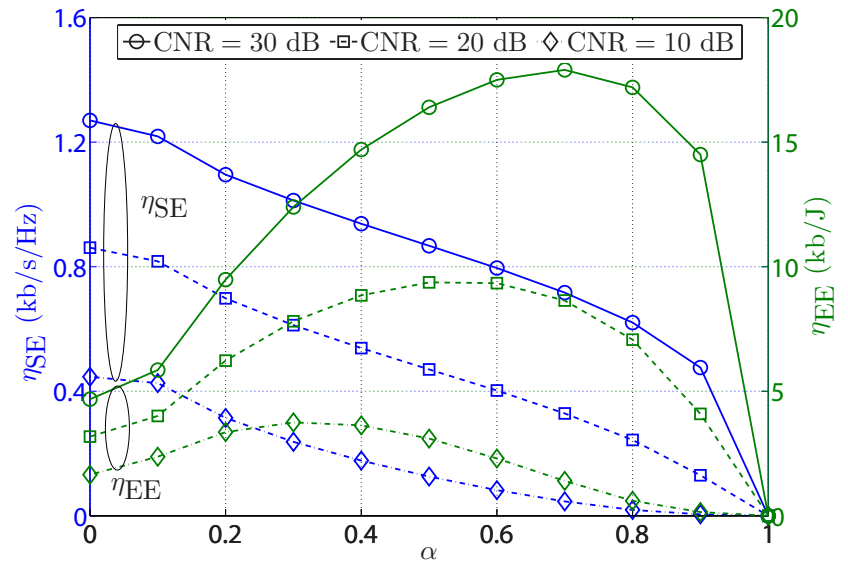

Fig. 4: EE and SE versus the priority parameter $\alpha$ for different CNR values.

of the previously mentioned functions and the MMSE. We observe that the optimal SE is more sensitive to MMSE than other functions. We can notice that channel estimators with an MMSE below 0.001 are required to achieve satisfactory results.

Simulation Example 4: In the last simulation example, we study the effect of the channel quality on the multiobjective optimization assuming MMSE $=0.001$ and $P_{T} / \theta_{\mathrm{P}}=0.11$. Fig. 4 plots the EE and SE versus $\alpha$ for different $\mathrm{CNR}$ values $(10 \mathrm{~dB}, 20 \mathrm{~dB}$ and $30 \mathrm{~dB})$. The SE curves have the same trend for different CNR values, with great improvement as CNR increases. Similarly, the EE curves improve with increasing the CNR; however, the maximum EE is attained at $\alpha=0.3,0.5$, and 0.7 for $\mathrm{CNR}=10 \mathrm{~dB}, 20 \mathrm{~dB}$ and $30 \mathrm{~dB}$, respectively. By comparing the relative improvement of the optimal SE and $\mathrm{EE}$, we observe that improving CNR gives more relative improvement to the optimal EE when compared to the optimal SE. Specifically, as the CNR increases from $10 \mathrm{~dB}$ to 30 $\mathrm{dB}$, the optimal EE improves by $60 \%$, while the optimal SE improves only by $20 \%$.

\section{CONCLUSION}

In this paper, we introduced a framework of multiobjective optimization to deal with the power loading problem for the OFDM systems with channel estimation error, considering the EE-SE trade-off. We proved that the multiobjective optimization of EE-SE is equivalent to the multiobjective problem that minimizes the total power consumption and maximizes the channel capacity. We explained how to select the trade-off parameter to switch between different practical communication scenarios, with different design requirements. Numerical results showed that low ratio of the maximum transmitted power budget to the total power consumption may significantly limit our objective functions performance, especially for the SE. To obtain satisfactory results, channel estimators with a maximum MMSE of $10^{-3}$ should be considered. The channel estimation error has a significant effect on the optimal EE and SE; it was observed that the later is more sensitive to the MMSE variations. On the hand, when the CNR increases the optimal EE achieves a better relative performance improvement than the optimal SE.

\section{REFERENCES}

[1] A. Fehske, G. Fettweis, J. Malmodin, and G. Biczok, "The global footprint of mobile communications: The ecological and economic perspective," IEEE Commun. Mag., vol. 49, no. 8, pp. 55-62, Aug. 2011.

[2] O. Amin, S. Bavarian, and L. Lampe, "Cooperative techniques for energy-efficient wireless communications," in Green Radio Communication Networks. Cambridge University Press, 2012, pp. 125-149.

[3] Y. Chen, S. Zhang, S. Xu, and G. Y. Li, "Fundamental trade-offs on green wireless networks," IEEE Commun. Mag., vol. 49, no. 6, pp. 30-37, Jun. 2011.

[4] C. Xiong, G. Y. Li, S. Zhang, Y. Chen, and S. Xu, "Energy-and spectralefficiency tradeoff in downlink OFDMA networks," IEEE Trans. Wireless Commun., vol. 10, no. 11, pp. 3874-3886, Nov. 2011.

[5] S. Huang, H. Chen, J. Cai, and F. Zhao, "Energy efficiency and spectralefficiency tradeoff in amplify-and-forward relay networks," IEEE Trans. Veh. Technol., to appear.

[6] O. Onireti, F. Heliot, and M. Imran, "On the energy efficiencyspectral efficiency trade-off of distributed MIMO systems," IEEE Trans. Commun., to appear.

[7] O. Amin and L. Lampe, "Opportunistic energy efficient cooperative communication," IEEE Wireless Commun. Lett., vol. 1, no. 5, pp. 412415, Oct. 2012.

[8] Y.-K. Chia, S. Sun, and R. Zhang, "Energy cooperation in cellular networks with renewable powered base stations," in Proc. IEEE Wireless Communications and Networking Conference, 2013, pp. 2542-2547.

[9] E. Bedeer, O. A. Dobre, M. H. Ahmed, and K. E. Baddour, "Joint optimization of bit and power loading for multicarrier systems," IEEE Wireless Commun. Lett., vol. 2, no. 4, pp. 447-450, Aug. 2013.

[10] R. Devarajan, S. C. Jha, U. Phuyal, and V. K. Bhargava, "Energyaware resource allocation for cooperative cellular network using multiobjective optimization approach," IEEE Trans. Commun., vol. 11, no. 5, pp. 1797-1807, May 2012.

[11] S. Ohno and G. B. Giannakis, "Capacity maximizing MMSE-optimal pilots for wireless OFDM over frequency-selective block Rayleighfading channels," IEEE Trans. Inf. Theory, vol. 50, no. 9, pp. 21382145, Sept. 2004.

[12] T. S. Rappaport, Wireless Communications: Principles and Practice. Prentice Hall PTR New Jersey, 1996.

[13] W. Dinkelbach, "On nonlinear fractional programming," Management Science, vol. 13, no. 7, pp. 492-498, 1967.

[14] M. Deruyck, E. Tanghe, W. Joseph, W. Vereecken, M. Pickavet, L. Martens, and B. Dhoedt, "Model for power consumption of wireless access networks," IET Science, Measurement and Technology, vol. 5, no. 4, pp. 155-161, Aug. 2011. 